\title{
On a problem of Janusz Matkowski and Jacek Wesołowski, II
}

\author{
JANusz MoraWIEC(D) AND Thomas ZÜRChER
}

Dedicated to Professor Karol Baron on the occasion of his 70th birthday.

Abstract. We continue our study started in Morawiec and Zürcher (Aequ Math 92(4):601$615,2018)$ of the functional equation

$$
\varphi(x)=\sum_{n=0}^{N} \varphi\left(f_{n}(x)\right)-\sum_{n=0}^{N} \varphi\left(f_{n}(0)\right)
$$

and its increasing and continuous solutions $\varphi:[0,1] \rightarrow[0,1]$ such that $\varphi(0)=0$ and $\varphi(1)=1$. In this paper we assume that $f_{0}, \ldots, f_{N}:[0,1] \rightarrow[0,1]$ are strictly increasing contractions such that

$$
0 \leq f_{0}(0)<f_{0}(1) \leq f_{1}(0)<\cdots<f_{N-1}(1) \leq f_{N}(0)<f_{N}(1) \leq 1
$$

and at least one of the weak inequalities is strong.

Mathematics Subject Classification. Primary 39B12, Secondary 37A05.

Keywords. Functional equations, Iterated function systems, Singular functions, Absolutely continuous functions.

\section{Introduction}

Fix $N \in \mathbb{N}$ and strictly increasing contractions $f_{0}, \ldots, f_{N}:[0,1] \rightarrow[0,1]$ such that

$$
0 \leq f_{0}(0)<f_{0}(1) \leq f_{1}(0)<\cdots<f_{N-1}(1) \leq f_{N}(0)<f_{N}(1) \leq 1 .
$$

We continue our study of the existence of solutions $\varphi$ of the functional equation

$$
\varphi(x)=\sum_{n=0}^{N} \varphi\left(f_{n}(x)\right)-\sum_{n=0}^{N} \varphi\left(f_{n}(0)\right)
$$


in the class $\mathcal{C}$ consisting of all increasing and continuous functions $\varphi:[0,1] \rightarrow$ $[0,1]$ satisfying the following boundary conditions

$$
\varphi(0)=0 \quad \text { and } \quad \varphi(1)=1 .
$$

In this paper we assume, in contrast to [11], that

$$
\bigcup_{n=0}^{N}\left[f_{n}(0), f_{n}(1)\right] \neq[0,1] \text {. }
$$

\section{Preliminaries}

Throughout this paper for all $k \in \mathbb{N}$ and $n_{1}, \ldots, n_{k} \in\{0, \ldots, N\}$ we denote the composition $f_{n_{1}} \circ \cdots \circ f_{n_{k}}$ by $f_{n_{1}, \ldots, n_{k}}$. Moreover, we extend the notation to the case $k=0$ by letting $f_{n_{1}, \ldots, n_{0}}$ be the identity.

We begin with three lemmas. The proof of the first one is very easy, so we omit it.

Lemma 2.1. Fix $m \in \mathbb{N}$ and nonnegative real numbers $\alpha_{1}, \ldots, \alpha_{m}$ such that $\sum_{i=1}^{m} \alpha_{i}=1$. If $\varphi_{1}, \ldots, \varphi_{m} \in \mathcal{C}$, then $\sum_{i=1}^{m} \alpha_{i} \varphi_{i} \in \mathcal{C}$.

Lemma 2.2. If $\varphi \in \mathcal{C}$, then $\varphi\left(f_{0}(0)\right)=0$ and $\varphi\left(f_{N}(1)\right)=1$.

Proof. By (2), (E), (1), and the monotonicity of $\varphi$ we have

$$
\begin{aligned}
1 & =\varphi(1)=\varphi\left(f_{N}(1)\right)-\varphi\left(f_{0}(0)\right)+\sum_{n=1}^{N}\left[\varphi\left(f_{n-1}(1)\right)-\varphi\left(f_{n}(0)\right)\right] \\
& \leq \varphi\left(f_{N}(1)\right)-\varphi\left(f_{0}(0)\right) .
\end{aligned}
$$

As the image of $[0,1]$ under $\varphi$ lies in $[0,1]$, we infer that $\varphi\left(f_{0}(0)\right)=0$ and $\varphi\left(f_{N}(1)\right)=1$.

Now we want to show that if all the contractions $f_{0}, \ldots, f_{N}$ are nonsingular (i.e. $f_{0}^{-1}(A), \ldots, f_{N}^{-1}(A)$ have Lebesgue measure zero for every set $A \subset[0,1]$ of Lebesgue measure zero, ${ }^{1}$ ) then the class $\mathcal{C}$ is determined by two of its subclasses $\mathcal{C}_{a}$ and $\mathcal{C}_{s}$ of all absolutely continuous and all singular functions, respectively. Repeating directly the proof of Remark 2.2 from [11] with the use of Lemma 2.2 we get the following result.

Lemma 2.3. Assume that all the contractions $f_{0}, \ldots, f_{N}$ are nonsingular. Then, both the absolutely continuous and the singular parts ${ }^{2}$ of every element from $\mathcal{C}$ satisfy $(\mathrm{E})$ for every $x \in[0,1]$.

\footnotetext{
${ }^{1}$ See [7]. Note also that as the inverses of the contractions exist and are continuous and increasing, being nonsingular is equivalent to the inverses being absolutely continuous, see for example Theorem 7.1.38 in [6].

${ }^{2}$ The parts are unique up to a constant. For definiteness, we choose them so that both of them map 0 to 0 .
} 
By the monotonicity of $f_{0}$ and $f_{N}$, it is easy to prove that the sequence $\left(f_{0}^{0, \ldots, 0}(0)\right)_{k \in \mathbb{N}}$ is increasing and the sequence $\left(f^{N, \ldots, N}(1)\right)_{k \in \mathbb{N}}$ is decreasing. Hence both are convergent. Put

$$
\mathbf{0}=\lim _{k \rightarrow \infty} f_{\underbrace{0, \ldots, 0}}(0) \text { and } \mathbf{1}=\lim _{k \rightarrow \infty} f_{\underbrace{N, \ldots, N}_{k}}^{(1) .}
$$

It is clear that $\mathbf{0}$ is the unique fixed point of $f_{0}$ and $\mathbf{1}$ is the unique fixed point of $f_{N}$, i.e.

$$
f_{0}(\mathbf{0})=\mathbf{0} \quad \text { and } \quad f_{N}(\mathbf{1})=\mathbf{1} .
$$

Moreover,

$$
\mathbf{1}=\lim _{k \rightarrow \infty} f_{\underbrace{N, \ldots, N}_{k}}^{(0),}
$$

because for every $k \in \mathbb{N}$ we have $\left|f_{\underbrace{N, \ldots, N}_{k}}^{(1)}-f_{\underbrace{N, \ldots, N}_{k}}(0)\right| \leq c^{k}$, where $c \in(0,1)$ is a Lipschitz constant of $f_{N}$.

Lemma 2.4. Assume that $\varphi \in \mathcal{C}$. Then $\varphi(\mathbf{0})=0$ and $\varphi(\mathbf{1})=1$.

Proof. We first prove that $\varphi(\mathbf{0})=0$.

By Lemma 2.2 we have $\varphi\left(f_{0}(0)\right)=0$. Fix $k \in \mathbb{N}$ and assume inductively that $\varphi(\underbrace{f_{0, \ldots, 0}}_{k}(0))=0$. Applying the induction hypothesis, $(\mathrm{E})$, Lemma 2.2 and the monotonicity of $f_{0}, \ldots, f_{N}$ and $\varphi$, we get

$$
\begin{aligned}
& 0=\varphi(\underbrace{f_{0, \ldots, 0}^{0, \ldots}}_{k}(0))=\sum_{n=0}^{N} \varphi\left(f_{n}\left(f^{0, \ldots, 0}(0)\right)\right)-\sum_{n=0}^{N} \varphi\left(f_{n}(0)\right) \\
& =\varphi(\underbrace{f_{0, \ldots, 0}(0)}_{k+1})+\sum_{n=1}^{N} \varphi\left(f_{n}\left(f_{0, \ldots, 0}^{0, \ldots)}\right)\right)-\sum_{n=1}^{N} \varphi\left(f_{n}(0)\right) \\
& \geq \varphi(\underbrace{f_{0, \ldots, 0}}_{k+1}(0)) \geq 0 .
\end{aligned}
$$

Hence $\varphi\left(f_{f_{0+1}^{0, \ldots, 0}}^{0}(0)\right)=0$. Now the continuity of $\varphi$ gives

$$
\varphi(\mathbf{0})=\lim _{k \rightarrow \infty} \varphi(\underbrace{f_{0, \ldots, 0}}_{k}(0))=0 .
$$

To prove that $\varphi(\mathbf{1})=1$ observe first that by (1) and the monotonicity of $\varphi$ we have $\varphi\left(f_{n}(1)\right) \leq \varphi\left(f_{n+1}(0)\right)$ for every $n \in\{0, \ldots, N-1\}$. We want to show that

$$
\varphi\left(f_{n}(1)\right)=\varphi\left(f_{n+1}(0)\right)
$$


for every $n \in\{0, \ldots, N-1\}$. Suppose that, contrary to our claim, there exists $n \in\{0, \ldots, N-1\}$ such that $\varphi\left(f_{n}(1)\right)<\varphi\left(f_{n+1}(0)\right)$. Then, using Lemma 2.2 and arguing as in its proof, we obtain

$$
\begin{aligned}
1 & =\varphi(1)=\sum_{n=0}^{N-1} \varphi\left(f_{n}(1)\right)+1-\sum_{n=0}^{N} \varphi\left(f_{n}(0)\right) \\
& <\sum_{n=0}^{N-1} \varphi\left(f_{n+1}(0)\right)+1-\sum_{n=0}^{N} \varphi\left(f_{n}(0)\right)=1-\varphi\left(f_{0}(0)\right)=1,
\end{aligned}
$$

a contradiction.

Now we show by induction that

$$
\varphi(\underbrace{f_{N, \ldots, N}}_{k}(1))=1
$$

for all $k \in \mathbb{N}$. The first step of the induction holds due to Lemma 2.2. Fix $k \in \mathbb{N}$ and assume that (6) holds. Then applying (6), (E), Lemma 2.2, (5) and the monotonicity of $f_{0}, \ldots, f_{N}$ and $\varphi$ we get

$$
\begin{aligned}
1 & =\varphi(\underbrace{f_{N, \ldots, N}}_{k}(1))=\sum_{n=0}^{N} \varphi\left(f_{n}\left(f_{f_{k}^{N, \ldots, N}}(1)\right)\right)-\sum_{n=1}^{N} \varphi\left(f_{n}(0)\right) \\
& \leq \sum_{n=0}^{N-1} \varphi\left(f_{n}\left(f_{f_{k}^{N, \ldots, N}}^{N}(1)\right)\right)+\varphi(\underbrace{f_{N, \ldots, N}^{N, \ldots}}_{k+1}(1))-\sum_{n=0}^{N-1} \varphi\left(f_{n}(1)\right) \\
& \leq \varphi(\underbrace{f_{N, \ldots, N}^{N, \ldots}}_{k+1}(1)) \leq 1 .
\end{aligned}
$$

Hence $\varphi(\underbrace{f_{N, \ldots, N}}_{k+1}(1))=1$. Finally, passing with $k$ to infinity in (6) and using the continuity of $\varphi$ we obtain $\varphi(\mathbf{1})=1$.

\section{Basic property of solutions}

Define recursively a sequence $\left(A_{k}\right)_{k \in \mathbb{N}}$ of subsets of the interval $[0,1]$ as follows:

$$
A_{0}=[0,1] \quad \text { and } \quad A_{k}=\bigcup_{n=0}^{N} f_{n}\left(A_{k-1}\right) \quad \text { for every } k \in \mathbb{N} .
$$

By (3) we have $A_{1}=\bigcup_{n=0}^{N}\left[f_{n}(0), f_{n}(1)\right] \varsubsetneqq A_{0}$. Moreover, a witness of the strict inclusion can be found that is different from 0 and 1 . This jointly with 
an easy induction shows that $A_{k+1} \varsubsetneqq A_{k}$ for every $k \in \mathbb{N}$. Again there is a witness of the strict inequality differing from 0 and 1 . Put

$$
A_{*}=\bigcap_{k \in \mathbb{N}} A_{k} .
$$

It is clear that $A_{*}$ is compact and

$$
A_{*}=\bigcup_{n=0}^{N} f_{n}\left(A_{*}\right)
$$

We will show that the just constructed set $A_{*}$, called the attractor of the iterated function system $\left\{f_{0}, \ldots, f_{N}\right\}$ (see [1]), is a Cantor-like set, i.e. uncountable, nowhere dense and a perfect subset of $\mathbb{R}$ (see [13]); note that $A_{*}$ is uncountable and nowhere dense, which follows from its construction. Moreover, we will see in Theorem 3.6 that $A_{*}$ is perfect and in Example 3.5 that it is of Lebesgue measure zero if $f_{0}, \ldots, f_{N}$ are similitudes, whereas in the general case it can happen that $A_{*}$ is of positive Lebesgue measure (see [10]).

From the construction we have

$$
A_{*}=\bigcap_{k \in \mathbb{N}}\left(\bigcup_{n_{1}, \ldots, n_{k} \in\{0, \ldots, N\}}\left[f_{n_{1}, \ldots, n_{k}}(0), f_{n_{1}, \ldots, n_{k}}(1)\right]\right) .
$$

Whenever a point $x$ can be written as

$$
x=\lim _{k \rightarrow \infty} f_{x_{1}, \ldots, x_{k}}(0)=\lim _{k \rightarrow \infty} f_{x_{1}, \ldots, x_{k}}(1),
$$

we say that $x$ has an address $^{3}$ (see [1]).

Lemma 3.1. The set $A_{*}$ is exactly the set of points in $[0,1]$ that have an address.

Proof. Let $x \in A_{*}$. Note that for every $k \in \mathbb{N}$ there exist $x_{1}^{k}, \ldots, x_{k}^{k} \in$ $\{0, \ldots, N\}$ such that $x \in\left[f_{x_{1}^{k}, \ldots, x_{k}^{k}}(0), f_{x_{1}^{k}, \ldots, x_{k}^{k}}(1)\right]$ with $x_{m}^{n}$ not necessarily agreeing with $x_{m}^{l}$ for different $l$ and $n$, however, as each $x_{m}^{l}$ is chosen from the finite set $\{0, \ldots, N\}$, we may apply a Cantor diagonal argument to get a sequence as wished.

It is easy to see that every sequence $\left(x_{k}\right)_{k \in \mathbb{N}}$ of elements of $\{0, \ldots, N\}$ is an address of a point from the set $A_{*}$.

Note that

$$
\mathbf{0}=\min A_{*} \quad \text { and } \quad \mathbf{1}=\max A_{*} .
$$

Since $A_{*}$ is a closed set, it follows that $[\mathbf{0}, \mathbf{1}] \backslash A_{*}$ is an open set. Moreover,

$$
[\mathbf{0}, \mathbf{1}] \backslash A_{*}=\bigcup_{k \in \mathbb{N}} \bigcup_{\substack{0 \leq n_{1}, \ldots, n_{k-1} \leq N \\ 0 \leq n_{k} \leq N-1}}\left(f_{n_{1}, \ldots, n_{k}}(\mathbf{1}), f_{n_{1}, \ldots, n_{k}+1}(\mathbf{0})\right)
$$

\footnotetext{
${ }^{3}$ We have come across the term coding as well.
} 
and for all $k \in \mathbb{N}, n_{1}, \ldots, n_{k-1} \in\{0, \ldots, N\}$ and $n_{k} \in\{0, \ldots, N-1\}$ the interval $\left(f_{n_{1}, \ldots, n_{k}}(\mathbf{1}), f_{n_{1}, \ldots, n_{k}+1}(\mathbf{0})\right)$ is a connected component of the set $[\mathbf{0}, \mathbf{1}] \backslash A_{*}$.

Now we are in a position to show that any $\varphi \in \mathcal{C}$ is constant on the closure of each connected component of the set $[0,1] \backslash A_{*}$. We do it in two steps.

Lemma 3.2. Assume that $\varphi \in \mathcal{C}$. Then:

(i) $\left.\varphi\right|_{[0,0]}=0$;

(ii) $\left.\varphi\right|_{[1,1]}=1$;

(iii) $\left.\varphi\right|_{\left[f_{n}(\mathbf{1}), f_{n+1}(\mathbf{0})\right]}$ is constant for every $n \in\{0, \ldots, N-1\}$.

Proof. To prove (i) and (ii) it is enough to apply Lemma 2.4 jointly with the monotonicity of $\varphi$.

Let us tackle (iii). According to (5) and to the monotonicity of $f_{0}, \ldots, f_{N}$ and $\varphi$, we see that $\varphi\left(f_{n}(\mathbf{1})\right) \leq \varphi\left(f_{n+1}(\mathbf{0})\right)$. Suppose that, contrary to our claim, there exists $n \in\{0, \ldots, N-1\}$ such that $\varphi\left(f_{n}(\mathbf{1})\right)<\varphi\left(f_{n+1}(\mathbf{0})\right)$. Then, using Lemma 2.4, (E), and the first equality of (4) we get

$$
\begin{aligned}
1 & =\varphi(\mathbf{1})=\sum_{n=0}^{N-1} \varphi\left(f_{n}(\mathbf{1})\right)+\varphi\left(f_{N}(\mathbf{1})\right)-\sum_{n=0}^{N} \varphi\left(f_{n}(0)\right) \\
& <\sum_{n=0}^{N-1} \varphi\left(f_{n+1}(\mathbf{0})\right)+1-\sum_{n=0}^{N} \varphi\left(f_{n}(0)\right)=\varphi(\mathbf{0})-\varphi\left(f_{0}(\mathbf{0})\right)+1=1,
\end{aligned}
$$

a contradiction.

Lemma 3.3. Assume that $\varphi \in \mathcal{C}$. Then for all $k \in \mathbb{N}, n_{1}, \ldots, n_{k-1} \in\{0, \ldots, N\}$ and $n_{k} \in\{0, \ldots, N-1\}$ there exists $c_{n_{1}, \ldots, n_{k}} \in[0,1]$ such that

$$
\left.\varphi\right|_{\left[f_{n_{1}, \ldots, n_{k}}(\mathbf{1}), f_{n_{1}, \ldots, n_{k}+1}(\mathbf{0})\right]}=c_{n_{1}, \ldots, n_{k}} .
$$

Proof. We proceed by induction on $k$.

The first step of the induction is implied by assertion (iii) of Lemma 3.2.

Fix $k \in \mathbb{N}, n_{1}, \ldots, n_{k-1} \in\{0, \ldots, N\}, n_{k} \in\{0, \ldots, N-1\}$ and assume that there exists $c_{n_{1}, \ldots, n_{k}} \in[0,1]$ such that (11) holds. Then (11), (E) and the monotonicity of $f_{0}, \ldots, f_{N}$ and $\varphi$ imply

$$
\begin{aligned}
c_{n_{1}, \ldots, n_{k}} & =\varphi\left(f_{n_{1}, \ldots, n_{k}}(\mathbf{1})\right)=\sum_{n=0}^{N} \varphi\left(f_{n, n_{1}, \ldots, n_{k}}(\mathbf{1})\right)-\sum_{n=0}^{N} \varphi\left(f_{n}(0)\right) \\
& \leq \sum_{n=0}^{N} \varphi\left(f_{n, n_{1}, \ldots, n_{k}+1}(\mathbf{0})\right)-\sum_{n=0}^{N} \varphi\left(f_{n}(0)\right)=\varphi\left(f_{n_{1}, \ldots, n_{k}+1}(\mathbf{0})\right) \\
& =c_{n_{1}, \ldots, n_{k}} .
\end{aligned}
$$

Hence

$$
\sum_{n=0}^{N} \varphi\left(f_{n, n_{1}, \ldots, n_{k}}(\mathbf{1})\right)=\sum_{n=0}^{N} \varphi\left(f_{n, n_{1}, \ldots, n_{k}+1}(\mathbf{0})\right),
$$


and applying again the monotonicity of $f_{0}, \ldots, f_{N}$ and $\varphi$, we obtain

$$
\varphi\left(f_{n, n_{1}, \ldots, n_{k}}(\mathbf{1})\right)=\varphi\left(f_{n, n_{1}, \ldots, n_{k}+1}(\mathbf{0})\right)
$$

for every $n \in\{0, \ldots, N\}$.

Combining Lemmas 3.2 and 3.3 with (10), we get the following result.

Theorem 3.4. If the set $A_{*}$ has Lebesgue measure zero, then $\mathcal{C}=\mathcal{C}_{s}$.

We now give an example of contractions $f_{0}, \ldots, f_{N}$ for which the set $A_{*}$ is of Lebesgue measure zero.

Example 3.5. Assume additionally to our assumptions in the introduction that $f_{0}, \ldots, f_{N}$ are similitudes, i.e.

$$
f_{n}(x)=\left(\beta_{n}-\alpha_{n}\right) x+\alpha_{n}
$$

for all $x \in[0,1]$ and $n \in\{0, \ldots, N\}$, where

$$
0 \leq \alpha_{0}<\beta_{0} \leq \alpha_{1}<\beta_{1} \leq \cdots \leq \alpha_{N}<\beta_{N} \leq 1 \text { and } \bigcup_{n=0}^{N}\left[\alpha_{n}, \beta_{n}\right] \neq[0,1] .
$$

Clearly, (1) and (3) hold. Denote by $l$ the Lebesgue measure on the real line and put $d=l\left(A_{0} \backslash A_{1}\right)$. By a simple induction we get $l\left(A_{k} \backslash A_{k+1}\right)=d(1-d)^{k}$ for every $k \in \mathbb{N}$. From (1) and (3) we infer that $d \in(0,1)$ and hence that

$$
l\left(A_{*}\right)=1-\sum_{k=0}^{\infty} l\left(A_{k} \backslash A_{k+1}\right)=1-\frac{d}{1-(1-d)}=0 .
$$

We finish this section with one more property of the set $A_{*}$.

Theorem 3.6. The set $A_{*}$ is perfect.

Proof. We know from its definition that $A_{*}$ is closed, and it is nonempty by (9).

Let $x \in A_{*}$ and fix an address of $x$, i.e. a sequence $\left(x_{k}\right)_{k \in \mathbb{N}}$ of elements of $\{0, \ldots, N\}$ satisfying (8); we can choose such a sequence according to Lemma 3.1. To complete the proof, we need to show that in each neighbourhood of $x$ we can find some element belonging to $A_{*} \backslash\{x\}$.

Fix $\varepsilon>0$ and $m \in \mathbb{N}$ so large that $L^{m-1}<\varepsilon$, where $L \in(0,1)$ is the largest Lipschitz constant of the given contractions $f_{0}, \ldots, f_{N}$. Define a sequence $\left(y_{k}\right)_{k \in \mathbb{N}}$ by putting $y_{k}=x_{k}$ for all $k \neq m$ and choosing arbitrarily $y_{m} \in\{0, \ldots, N\} \backslash\left\{x_{m}\right\}$. Then

$$
y=\lim _{k \rightarrow \infty} f_{y_{1}, \ldots, y_{k}}(0) \in A_{*} .
$$

Since all considered contractions are injective and the addresses of points $x$ and $y$ differ only in the $m$-th coordinate, it follows that $y \neq x$. Moreover,

$$
\begin{aligned}
|x-y| & =\lim _{k \rightarrow \infty}\left|f_{x_{1}, \ldots, x_{m-1}}\left(f_{x_{m}, \ldots, x_{k}}(0)\right)-f_{x_{1}, \ldots, x_{m-1}}\left(f_{y_{m}, \ldots, y_{k}}(0)\right)\right| \\
& \leq L^{m-1} \lim _{k \rightarrow \infty}\left|f_{x_{m}, \ldots, x_{k}}(0)-f_{y_{m}, \ldots, y_{k}}(0)\right| \leq L^{m-1}<\varepsilon .
\end{aligned}
$$


The proof is complete.

\section{Existence of solutions}

In the previous section we have discussed the behaviour of functions belonging to the class $\mathcal{C}$, but, up to now, we do not know if $\mathcal{C}$ contains any function at all. In this section, we want to show that $\mathcal{C} \neq \emptyset$.

Fix positive real numbers $p_{0}, \ldots, p_{N}$ such that

$$
\sum_{n=0}^{N} p_{n}=1 \text {. }
$$

Then there exists a unique Borel probability measure $\mu$ such that

$$
\mu(B)=\sum_{n=0}^{N} p_{n} \mu\left(f_{n}^{-1}(B)\right)
$$

for every Borel set $B \subset[0,1]$ (see [5]; cf. [4]). From now on the letter $\mu$ will be reserved for the unique Borel probability measure satisfying (13) for every Borel set $B \subset[0,1]$.

Now we are interested in some properties of the measure $\mu$ that will be needed later. We begin with a well-known folklore lemma; for its proof the reader can consult [8].

Lemma 4.1. The measure $\mu$ is either singular or absolutely continuous with respect to the Lebesgue measure on $\mathbb{R}$.

To formulate the next lemma, which is also well-known (see e.g. [1]), we recall that the support of the measure $\mu$ is the set $\operatorname{supp} \mu$ of all points $x \in[0,1]$ such that $\mu([x-\varepsilon, x+\varepsilon])>0$ for every $\varepsilon>0$.

Lemma 4.2. We have supp $\mu=A_{*}$. In particular, $\mu\left([0,1] \backslash A_{*}\right)=0$.

Lemma 4.3. The measure $\mu$ is continuous.

Proof. To prove that $\mu$ is continuous it is enough to show $\mu(\{x\})=0$ for every $x \in[0,1]$.

Fix $x \in[0,1]$.

If $x \notin A_{*}$, then $\mu(\{x\})=0$ by Lemma 4.2 , hence we assume now that $x \in A_{*}$ and choose an address of $x$, that is a sequence $\left(x_{k}\right)_{k \in \mathbb{N}}$ of elements of $\{0, \ldots, N\}$ such that $(8)$ holds. Note that the monotonicity of $f_{0}, \ldots, f_{N}$ implies

$$
f_{x_{1}, \ldots, x_{k}}(0) \leq f_{x_{1}, \ldots, x_{k+1}}(0) \leq x \leq f_{x_{1}, \ldots, x_{k+1}}(1) \leq f_{x_{1}, \ldots, x_{k}}(1)
$$

for every $k \in \mathbb{N}$. 
First we want to prove that

$$
\mu\left(\left\{f_{n}(0)\right\}\right)=\mu\left(\left\{f_{n}(1)\right\}\right)=0
$$

for every $n \in\{0, \ldots, N\}$.

We begin with proving that

$$
\mu(\{0\})=\mu(\{1\})=0 .
$$

If $f_{0}(0)>0$, then $(13)$ gives

$$
\mu(\{0\})=\sum_{n=0}^{N} p_{n} \mu\left(\left\{f_{n}^{-1}(0)\right\}\right)=\sum_{n=0}^{N} p_{n} \mu(\emptyset)=0 .
$$

If $f_{0}(0)=0$, then $(13)$ yields

$$
\mu(\{0\})=\sum_{n=0}^{N} p_{n} \mu\left(\left\{f_{n}^{-1}(0)\right\}\right)=p_{0} \mu(\{0\})+\sum_{n=1}^{N} p_{n} \mu(\emptyset)=p_{0} \mu(\{0\}),
$$

and since $p_{0} \in(0,1)$ we conclude that $\mu(\{0\})=0$.

In the same way, considering two cases $\left(f_{N}(1)<1\right.$ and $\left.f_{N}(1)=1\right)$ and using (13) jointly with the fact that $p_{N} \in(0,1)$ in the second case, we get $\mu(\{1\})=0$.

Using (13), (1) and (16) we obtain

$$
\begin{aligned}
& \mu\left(\left\{f_{0}(0)\right\}\right)=\sum_{n=0}^{N} p_{n} \mu\left(\left\{f_{n}^{-1}\left(f_{0}(0)\right)\right\}\right)=p_{0} \mu(\{0\})=0, \\
& \mu\left(\left\{f_{N}(1)\right\}\right)=\sum_{n=0}^{N} p_{n} \mu\left(\left\{f_{n}^{-1}\left(f_{N}(1)\right)\right\}\right)=p_{N} \mu(\{1\})=0
\end{aligned}
$$

and

$$
\begin{aligned}
\mu\left(\left\{f_{m}(1), f_{m+1}(0)\right\}\right) & =\sum_{n=0}^{N} p_{n} \mu\left(\left\{f_{n}^{-1}\left(f_{m}(1)\right), f_{n}^{-1}\left(f_{m+1}(0)\right)\right\}\right) \\
& \leq 2\left(p_{m} \mu(\{1\})+p_{m+1} \mu(\{0\})\right)=0
\end{aligned}
$$

for every $m \in\{0, \ldots, N-1\}$.

By (15) and (1), equality (13) implies

$$
\mu\left(f_{n}(B)\right)=p_{n} \mu(B)
$$

for all $n \in\{0, \ldots, N\}$ and Borel sets $B \subset[0,1]$. 
Finally, applying (14) and equality (17) $k$ times jointly with the fact that $\mu$ is a probability measure we get

$$
\begin{aligned}
\mu(\{x\}) & =\mu\left(\bigcap_{k \in \mathbb{N}}\left[f_{x_{1}, \ldots, x_{k}}(0), f_{x_{1}, \ldots, x_{k}}(1)\right]\right) \\
& =\lim _{k \rightarrow \infty} \mu\left(\left[f_{x_{1}, \ldots, x_{k}}(0), f_{x_{1}, \ldots, x_{k}}(1)\right]\right) \\
& =\lim _{k \rightarrow \infty} \prod_{i=1}^{k} p_{x_{i}} \leq \lim _{k \rightarrow \infty}\left(\max \left\{p_{0}, \ldots, p_{N}\right\}\right)^{k}=0 .
\end{aligned}
$$

The proof is complete.

Combining Lemmas 4.2 and 4.3 with (10) we get the following corollary.

Corollary 4.4. The measure $\mu$ vanishes on each of the intervals: $[0, \mathbf{0}],[\mathbf{1}, 1]$, $\left[f_{n_{1}, \ldots, n_{k}}(\mathbf{1}), f_{n_{1}, \ldots, n_{k}+1}(\mathbf{0})\right]$ with $k \in \mathbb{N}, n_{1}, \ldots, n_{k-1} \in\{0, \ldots, N\}$ and $n_{k} \in$ $\{0, \ldots, N-1\}$.

Define the function $\varphi:[0,1] \rightarrow[0,1]$ by

$$
\varphi(x)=\mu([0, x]) .
$$

From now on the letter $\varphi$ will be reserved for the just defined function.

Repeating the proof of Theorem 3.3 from [11] we get the following result.

Theorem 4.5. Either $\varphi \in \mathcal{C}_{a}$ or $\varphi \in \mathcal{C}_{s}$.

As a consequence of Theorem 4.5, we have $\varphi \in \mathcal{C}$. Lemma 4.2 implies that $\varphi$ cannot be constant on an open interval having nonempty intersection with the attractor $A_{*}$. Therefore, all the constants $c_{n_{1}, \ldots, n_{k}}$ occurring in the assertion of Lemma 3.3 (associated with the above constructed $\varphi$ ) are pairwise different and belong to the open interval $(0,1)$.

We finish this section by giving a precise formula for $\varphi$.

Theorem 4.6. Assume that $x \in[0,1]$.

(i) If $x \in[0, \mathbf{0}]$, then $\varphi(x)=0$.

(ii) If $x \in[\mathbf{1}, 1]$, then $\varphi(x)=1$.

(iii) If $x \in A_{*}$ and $\left(x_{l}\right)_{l \in \mathbb{N}}$ is an address of $x$, then

$$
\varphi(x)=\sum_{l=1}^{\infty} \operatorname{sgn}\left(x_{l}\right)\left[\prod_{n=0}^{N} p_{n}^{\#\left\{i \in\{1, \ldots, l-1\}: x_{i}=n\right\}} \cdot \sum_{n=0}^{x_{l}-1} p_{n}\right] .
$$


(iv) If $x \in\left[f_{x_{1}, \ldots, x_{k}}(\mathbf{1}), f_{x_{1}, \ldots, x_{k}+1}(\mathbf{0})\right]$ with $k \in \mathbb{N}, x_{1}, \ldots, x_{k-1} \in\{0, \ldots, N\}$ and $x_{k} \in\{0, \ldots, N-1\}$, then

$$
\begin{aligned}
\varphi(x) & =\sum_{l=1}^{k} \operatorname{sgn}\left(x_{l}\right)\left[\prod_{n=0}^{N} p_{n}^{\#\left\{i \in\{1, \ldots, l-1\}: x_{i}=n\right\}} \cdot \sum_{n=0}^{x_{l}-1} p_{n}\right] \\
& +\prod_{n=0}^{N} p_{n}^{\#\left\{i \in\{1, \ldots, k\}: x_{i}=n\right\}} .
\end{aligned}
$$

Proof. Assertions (i) and (ii) are trivially implied by assertions (i) and (ii) of Lemma 3.2. The proof of assertion (iii) follows very closely the proof of Theorem 3.6 from [11], so we omit it. Assertion (iv) is a consequence of Lemma 3.3, the fact that $\left(x_{1}, \ldots, x_{k}, N, \ldots\right)$ is the address of the point $f_{x_{1}, \ldots, x_{k}}(\mathbf{1})$ and assertion (iii); indeed

$$
\begin{aligned}
\varphi(x)= & \varphi\left(f_{x_{1}, \ldots, x_{k}}(\mathbf{1})\right)=\sum_{l=1}^{k} \operatorname{sgn}\left(x_{l}\right)\left[\prod_{n=0}^{N} p_{n}^{\#\left\{i \in\{1, \ldots, l-1\}: x_{i}=n\right\}} \cdot \sum_{n=0}^{x_{l}-1} p_{n}\right] \\
& +\sum_{l=k+1}^{\infty}\left[\prod_{n=0}^{N} p_{n}^{\#\left\{i \in\{1, \ldots, k\}: x_{i}=n\right\}} p_{N}^{l-k-1}\right] \cdot\left(1-p_{N}\right) \\
= & \sum_{l=1}^{k} \operatorname{sgn}\left(x_{l}\right)\left[\prod_{n=0}^{N} p_{n}^{\#\left\{i \in\{1, \ldots, l-1\}: x_{i}=n\right\}} \cdot \sum_{n=0}^{x_{l}-1} p_{n}\right] \\
& +\prod_{n=0}^{N} p_{n}^{\#\left\{i \in\{1, \ldots, k\}: x_{i}=n\right\}} .
\end{aligned}
$$

This finishes the proof.

\section{More about the class $\mathcal{C}$}

As we have seen in Theorem 4.5 , with each sequence $\left(p_{0}, \ldots, p_{N}\right)$ of positive real numbers satisfying (12) we have associated a continuous increasing surjective solution $\varphi_{p_{0}, \ldots, p_{N}}:[0,1] \rightarrow[0,1]$ of Eq. $(\mathrm{E})$. We denote by $\mathcal{W}$ the set of all these solutions. The main purpose of this section is to prove the following result.

Theorem 5.1. The set $\mathcal{W}$ is linearly independent and its convex hull is contained in $\mathcal{C}$.

\subsection{Proof of Theorem 5.1}

The statement concerning the convex hull follows from Lemma 2.1. 
The proof of the independence will be divided into several lemmas. Before we formulate the first one, note that for every $y \in A_{*}$ equality (7) guarantees that there exists at least one $n \in\{0, \ldots, N\}$ such that $y \in f_{n}\left(A_{*}\right)$. Therefore, we can define a transformation $T: A_{*} \rightarrow[0,1]$ by putting

$$
T(y)=f_{n(y)}^{-1}(y),
$$

where

$$
n(y)=\max \left\{n \in\{0, \ldots, N\}: y \in f_{n}\left(A_{*}\right)\right\} .
$$

Lemma 5.2. The transformation $T$ maps $A_{*}$ into $A_{*}$ and it is measure preserving for $\mu$.

Proof. To see that $T\left(A_{*}\right) \subset A_{*}$ we fix $y \in A_{*}$. Then the injectivity of $f_{n(y)}$ implies that there is exactly one $x \in A_{*}$ such that $y=f_{n(y)}(x)$. Thus $T(y)=$ $x \in A_{*}$.

Now we prove that $T$ is measure preserving for $\mu$.

Fix a Borel set $B \subset A_{*}$. As $A_{*} \subset \bigcup_{n=0}^{N}\left[f_{n}(0), f_{n}(1)\right]$, we have

$$
T^{-1}(B)=\bigcup_{n=0}^{N}\left\{y \in\left[f_{n}(0), f_{n}(1)\right] \cap A_{*}: T(y) \in B\right\} .
$$

Then using Lemma 4.3 jointly with (1) and the fact that the set $\bigcup_{i=0}^{N} f_{i}^{-1}(y)$ contains just one element in the case where $y \in\left(f_{n}(0), f_{n}(1)\right)$ and at most two elements in the case where $y \in\left\{f_{n}(0), f_{n}(1)\right\}$, we obtain

$$
\begin{aligned}
\mu\left(T^{-1}(B)\right) & =\sum_{n=0}^{N} \mu\left(\left\{y \in\left[f_{n}(0), f_{n}(1)\right] \cap A_{*}: T(y) \in B\right\}\right) \\
& =\sum_{n=0}^{N} \mu\left(\left\{y \in\left[f_{n}(0), f_{n}(1)\right] \cap A_{*}: f_{n}^{-1}(y) \in B\right\}\right) \\
& =\sum_{n=0}^{N} \mu\left(\left\{y \in\left[f_{n}(0), f_{n}(1)\right] \cap A_{*}: y \in f_{n}(B)\right\}\right) .
\end{aligned}
$$

Next note that $f_{n}(B) \subset f_{n}\left(A_{*}\right) \subset A_{*}$ for every $n \in\{0, \ldots, N\}$. Thus

$$
\mu\left(T^{-1}(B)\right)=\sum_{n=0}^{N} \mu\left(f_{n}(B)\right) .
$$

Finally, according to (17) we conclude that

$$
\mu\left(T^{-1}(B)\right)=\sum_{n=0}^{N} p_{n} \mu(B)=\mu(B),
$$

and the proof is complete. 
By Lemma 3.1 the points in $A_{*}$ are exactly the ones that have an address. The next lemma shows that we might run into slight problems with the uniqueness of the addresses if

$$
f_{0}(0)=0, \quad f_{N}(1)=1 \quad \text { and } \quad N_{b} \neq \emptyset,
$$

where

$$
N_{b}=\left\{n \in\{0, \ldots, N-1\}: f_{n}(1)=f_{n+1}(0)\right\} .
$$

\section{Lemma 5.3.}

(i) Every point from $A_{*}$ has at most two addresses, and if a point from $A_{*}$ has two addresses, then (18) holds and exactly one of the addresses belongs to the set

$Z_{b}=\left\{\left(x_{k}\right)_{k \in \mathbb{N}} \in\{0, \ldots, N\}^{\mathbb{N}}: \exists_{n \in \mathbb{N}}\left(x_{n} \in N_{b}\right.\right.$ and $x_{k}=N$ for every $\left.\left.k>n\right)\right\}$.

(ii) If (18) holds and a point from $A_{*}$ has an address belonging to the set $Z_{b}$, then it also has an address not belonging to the set $Z_{b}$.

(iii) Every point from $A_{*}$ has exactly one address if and only if (18) does not hold.

Proof. (i) Assume that $\left(x_{k}\right)_{k \in \mathbb{N}}$ and $\left(y_{k}\right)_{k \in \mathbb{N}}$ are two different addresses of a point $x \in A_{*}$. Put

$$
m=\min \left\{k \in \mathbb{N}: x_{k} \neq y_{k}\right\}
$$

and let $x_{m}<y_{m}$. Then according to (8) and (1) we have

$$
\begin{aligned}
x & =f_{x_{1}, \ldots, x_{m}}\left(\lim _{k \rightarrow \infty} f_{x_{m+1}, \ldots, x_{k}}(1)\right) \\
& \leq f_{x_{1}, \ldots, x_{m}}(1) \leq f_{y_{1}, \ldots, y_{m}}(0) \leq f_{y_{1}, \ldots, y_{m}}\left(\lim _{k \rightarrow \infty} f_{y_{m+1}, \ldots, y_{k}}(0)\right)=x .
\end{aligned}
$$

Thus $f_{x_{1}, \ldots, x_{m}}(1)=f_{y_{1}, \ldots, y_{m}}(0)$, and hence $f_{x_{m}}(1)=f_{y_{m}}(0) \in A_{*}$. Finally, making use of (1), we conclude that $x_{m} \in N_{b}, f_{0}(0)=0$ and $f_{N}(1)=1$. In consequence (18) holds, $\left(x_{k}\right)_{k \in \mathbb{N}} \in Z_{b}$ and $\left(y_{k}\right)_{k \in \mathbb{N}} \notin Z_{b}$. Moreover, if we assumed that $x$ has a third address $\left(z_{k}\right)_{k \in \mathbb{N}}$, different from both of the first ones, we would have $\left(z_{k}\right)_{k \in \mathbb{N}} \in Z_{b} \backslash\left\{\left(x_{k}\right)_{k \in \mathbb{N}}\right\}$, which is impossible.

(ii) Assume that (18) holds and let a point $x \in A_{*}$ has an address $\left(x_{k}\right)_{k \in \mathbb{N}} \in$ $Z_{b}$. Then there is $m \in N$ such that $x_{m} \in N_{b}$ and $x=f_{x_{1}, \ldots, x_{m}}(\mathbf{1})$. Applying now (18) we get

$$
x=f_{x_{1}, \ldots, x_{m}}(1)=f_{x_{1}, \ldots, x_{m}+1}(0)=f_{x_{1}, \ldots, x_{m}+1}(\mathbf{0}),
$$

which shows that (8) has an address not belonging to the set $Z_{b}$.

(iii) Assertion (i) implies that if (18) does not hold, then every point from $A_{*}$ has exactly one address.

Assume now that every point from $A_{*}$ has exactly one address and suppose that, on the contrary, (18) holds. Then $0=\mathbf{0}$ and $1=\mathbf{1}$. Fix $n \in N_{b}$ and 
put $x=f_{n}(\mathbf{1})$. Then $x \in A_{*}$ and $f_{n}(\mathbf{1})=f_{n}(1)=f_{n+1}(0)=f_{n+1}(\mathbf{0})$, which jointly with (8) implies that $x$ has two different addresses, a contradiction.

We now define a map $\pi:\{0, \ldots, N\}^{\mathbb{N}} \backslash Z_{b} \rightarrow A_{*}$ by putting

$$
\pi\left(\left(x_{k}\right)_{k \in \mathbb{N}}\right)=\lim _{k \rightarrow \infty} f_{x_{1}, \ldots, x_{k}}(0),
$$

where $Z_{b}$ is as in Lemma 5.3 in the case where (18) holds and $Z_{b}=\emptyset$ in the case where (18) does not hold.

Lemma 5.4. The map $\pi$ is a bijection.

Proof. It is enough to apply Lemma 5.3 and [1, Theorem 1 in Chapter 4.2].

Denote by $\sigma$ the Bernoulli shift, i.e. the map from $\{0, \ldots, N\}^{\mathbb{N}}$ into itself defined by

$$
\sigma\left(\left(x_{k}\right)_{k \in \mathbb{N}}\right)=\left(x_{k+1}\right)_{k \in \mathbb{N}} .
$$

Lemma 5.5. For every $n \in \mathbb{N}$ we have

$$
\sigma^{-n} \circ \pi^{-1}=\pi^{-1} \circ T^{-n} .
$$

Proof. We begin with proving that we have

$$
\pi \circ \sigma=T \circ \pi
$$

on $\{0, \ldots, N\}^{\mathbb{N}} \backslash Z_{b}$.

First, we note that $\sigma\left(\{0, \ldots, N\}^{\mathbb{N}} \backslash Z_{b}\right) \subset\{0, \ldots, N\}^{\mathbb{N}} \backslash Z_{b}$. Fix $\left(x_{k}\right)_{k \in \mathbb{N}} \in$ $\{0, \ldots, N\}^{\mathbb{N}} \backslash Z_{b}$ and put $z=\lim _{k \rightarrow \infty} f_{x_{2}, \ldots, x_{k}}(0)$. Then

$$
\pi\left(\sigma\left(\left(x_{k}\right)_{k \in \mathbb{N}}\right)\right)=\lim _{k \rightarrow \infty} f_{x_{2}, \ldots, x_{k}}(0)=z,
$$

and

$$
T\left(\pi\left(\left(x_{k}\right)_{k \in \mathbb{N}}\right)\right)=T\left(\lim _{k \rightarrow \infty} f_{x_{1}, \ldots, x_{k}}(0)\right)=T\left(f_{x_{1}}(z)\right)=f_{n\left(f_{x_{1}}(z)\right)}^{-1}\left(f_{x_{1}}(z)\right) .
$$

Since $z \in A_{*}$, we have $f_{x_{1}}(z) \in f_{x_{1}}\left(A_{*}\right)$, and so

$$
x_{1} \leq n\left(f_{x_{1}}(z)\right) \text {. }
$$

Suppose for a contradiction that $x_{1}<n\left(f_{x_{1}}(z)\right)$. Then $f_{x_{1}}(z) \in f_{x_{1}+1}\left(A_{*}\right)$, and by (1) we have $z=1$. Therefore, $f_{x_{1}}(1)=f_{x_{1}+1}(0)$ and $x_{k}=N$ for every $k \geq 2$, which is impossible as $\left(x_{k}\right)_{k \in \mathbb{N}} \notin Z_{b}$. In consequence $x_{1}=n\left(f_{x_{1}}(z)\right)$. Hence $f_{n\left(f_{x_{1}}(z)\right)}^{-1}\left(f_{x_{1}}(z)\right)=z$, which yields that (19) holds.

To complete the proof it is enough to proceed by induction with the use of (19).

Let us consider now the measure $\mathbb{P}_{p_{0}, \ldots, p_{N}}$ defined on $\{0, \ldots, N\}$ by

$$
\mathbb{P}_{p_{0}, \ldots, p_{N}}(\{k\})=p_{k} .
$$

Note that $\mathbb{P}_{p_{0}, \ldots, p_{N}}$ is a probability measure by (12). Further, we let $\mathbb{P}$ be the product measure on $\{0, \ldots, N\}^{\mathbb{N}}$ of $\mathbb{N}$ copies of the measure $\mathbb{P}_{p_{0}, \ldots, p_{N}}$. It is 
known that the Bernoulli shift is strong-mixing for $\mathbb{P}$ (see e.g. [2, Problem 4.3] or [3, Exercise 2.7.9]).

Lemma 5.6. If $B \subset\{0, \ldots, N\}^{\mathbb{N}} \backslash Z_{b}$ is a Borel set, then $\pi(B)$ is a Borel set and

$$
\mathbb{P}(B)=\mu(\pi(B)) .
$$

Proof. We first prove that if $B \subset\{0, \ldots, N\}^{\mathbb{N}} \backslash Z_{b}$ is a Borel set, then $\pi(B)$ is a Borel set as well.

As every Borel set in $\{0, \ldots, N\}^{\mathbb{N}} \backslash Z_{b}$ is generated by sets of the form

$$
B=\left(\left\{x_{1}\right\} \times \cdots \times\left\{x_{m}\right\} \times\{0, \ldots, N\}^{\mathbb{N}}\right) \backslash Z_{b},
$$

where $m \in \mathbb{N}$ and $x_{1}, \ldots, x_{m} \in\{0, \ldots, N\}$, it is sufficient to show that $\pi(B)$ is a Borel set for every set of the form (21).

Fix a set $B$ of the form (21) with $m \in \mathbb{N}$ and $x_{1}, \ldots, x_{m} \in\{0, \ldots, N\}$. If either (18) does not hold or (18) holds and $x_{m} \notin N_{b}$, then by Lemma 5.4 we have

$$
\pi(B)=f_{x_{1}, \ldots, x_{m}}\left(\pi\left(\{0, \ldots, N\}^{\mathbb{N}} \backslash Z_{b}\right)\right)=f_{x_{1}, \ldots, x_{m}}\left(A_{*}\right) .
$$

If (18) holds and $x_{m} \in N_{b}$, then by Lemma 5.4 we have

$$
\begin{aligned}
\pi(B) & =f_{x_{1}, \ldots, x_{m}}\left(\pi\left(\left(\{0, \ldots, N\}^{\mathbb{N}} \backslash Z_{b}\right) \backslash\{N\}^{\mathbb{N}}\right)\right) \\
& =f_{x_{1}, \ldots, x_{m}}\left(A_{*}\right) \backslash f_{x_{1}, \ldots, x_{m}}(\{\mathbf{1}\}) .
\end{aligned}
$$

Since $A_{*}$ and $\{\mathbf{1}\}$ are compact sets and $f_{0}, \ldots, f_{N}$ are contractions, it follows that $f_{x_{1}, \ldots, x_{m}}\left(A_{*}\right)$ and $f_{x_{1}, \ldots, x_{m}}(\{\mathbf{1}\})$ are compact sets. In consequence, we see that in both of the considered cases the set $\pi(B)$ is Borel.

Now we prove that (20) holds for every Borel set $B \subset\{0, \ldots, N\}^{\mathbb{N}} \backslash Z_{b}$.

Since every two Borel probability measures defined on $\{0, \ldots, N\}^{\mathbb{N}} \backslash Z_{b}$ agreeing on cylinders are equal, it suffices to show that (20) holds for every cylinder $B \subset\{0, \ldots, N\}^{\mathbb{N}} \backslash Z_{b}$. Moreover, by the additivity of the measures, we only need to show that (20) holds for every set of the form (21), where $m \in \mathbb{N}$ and $x_{1}, \ldots, x_{m} \in\{0, \ldots, N\}$.

Fix a set $B$ of the form (21) with $m \in \mathbb{N}$ and $x_{1}, \ldots, x_{m} \in\{0, \ldots, N\}$. Then either (22) or (23) is satisfied, and by Lemmas 4.3 and 4.2 we see that in both cases we have

$$
\mu\left(f_{x_{1}, \ldots, x_{m}}^{-1}(\pi(B))\right)=\mu\left(A_{*}\right)=1 .
$$

This jointly with (17) yields

$$
\mu(\pi(B))=\mu\left(f_{x_{1}}\left(\cdots\left(f_{x_{m}}\left(f_{x_{1}, \ldots, x_{m}}^{-1}(\pi(B))\right)\right) \cdots\right)\right)=\prod_{i=1}^{m} p_{x_{i}} .
$$

Finally, note that $\mathbb{P}(B)=\prod_{i=1}^{m} p_{x_{i}}$.

Lemma 5.7. The transformation $T$ is strong-mixing for $\mu$. 
Proof. The transformation $T$ is measure preserving for $\mu$ by Lemma 5.2. To prove that it is strong-mixing for $\mu$, fix two Borel sets $A, B \subset A_{*}$. Then using Lemmas 5.4 and 5.6, the fact that the Bernoulli shift is strong-mixing for $\mathbb{P}$ and Lemma 5.5 we get

$$
\begin{aligned}
\mu(A) \mu(B) & \left.=\mu\left(\pi\left(\pi^{-1}(A)\right)\right) \mu\left(\pi^{-1}(B)\right)\right)=\mathbb{P}\left(\pi^{-1}(A)\right) \mathbb{P}\left(\pi^{-1}(B)\right) \\
& =\lim _{m \rightarrow \infty} \mathbb{P}\left(\sigma^{-m}\left(\pi^{-1}(A)\right) \cap \pi^{-1}(B)\right)=\lim _{m \rightarrow \infty} \mathbb{P}\left(\pi^{-1}\left(T^{-m}(A)\right) \cap \pi^{-1}(B)\right) \\
& =\lim _{m \rightarrow \infty} \mathbb{P}\left(\pi^{-1}\left(T^{-m}(A) \cap B\right)\right)=\lim _{m \rightarrow \infty} \mu\left(T^{-m}(A) \cap B\right) .
\end{aligned}
$$

The proof is complete.

Denote by $\mathcal{M}^{T}\left(A_{*}\right)$ the set of all Borel probability measures defined on the $\sigma$-algebra of all Borel subsets of the interval $[0,1]$ supported on $A_{*}$, making the transformation $T$ measure preserving. Note that $\mu \in \mathcal{M}^{T}\left(A_{*}\right)$ by Lemma 4.2.

Lemma 5.8. Every family of pairwise mutually singular measures belonging to the set $\mathcal{M}^{T}\left(A_{*}\right)$ is linearly independent.

Proof. Fix $m \in \mathbb{N} \backslash\{1\}$, pairwise mutually singular measures $\mu_{1}, \ldots, \mu_{m} \in$ $\mathcal{M}^{T}\left(A_{*}\right)$, numbers $\alpha_{1}, \ldots, \alpha_{m} \in \mathbb{R} \backslash\{0\}$ and assume by contradiction that

$$
\sum_{i=1}^{m} \alpha_{i} \mu_{i}=0
$$

Since the measures are mutually singular, for each $i, j \in\{1, \ldots, m\}$ with $i \neq j$ there are sets $A_{i}^{j}$ and $A_{j}^{i}=X \backslash A_{i}^{j}$ such that $\mu_{i}\left(A_{i}^{i}\right)=\mu_{j}\left(A_{j}^{i}\right)=0$. Put $A_{m}=$ $\bigcup_{i=1}^{m-1} A_{m}^{i}$. Then

$$
0 \leq \mu_{m}\left(A_{m}\right)=\mu_{m}\left(\bigcup_{i=1}^{m-1} A_{m}^{i}\right) \leq \sum_{i=1}^{m-1} \mu_{m}\left(A_{m}^{i}\right)=0
$$

and for every $j \in\{1, \ldots, m-1\}$ we have

$$
1 \geq \mu_{j}\left(A_{m}\right)=\mu_{j}\left(X \backslash \bigcap_{i=1}^{m-1} A_{i}^{m}\right) \geq \mu_{j}\left(X \backslash A_{j}^{m}\right) \geq \mu_{j}(X)-\mu_{j}\left(A_{j}^{m}\right)=1 .
$$

In consequence

$$
\alpha_{m}=\sum_{i=1}^{m} \alpha_{i} \mu_{i}\left(X \backslash A_{m}\right)=0,
$$

and the proof is complete.

Now we are in a position to prove that the set $\mathcal{W}$ is linearly independent.

Fix $m$ different functions from $\mathcal{W}$ and consider the corresponding measures $\mu_{1}, \ldots, \mu_{m} \in \mathcal{M}^{T}\left(A_{*}\right)$. From Lemma 5.7 we infer that the transformation $T$ is ergodic for all the measures. Thus $\mu_{1}, \ldots, \mu_{m}$ are extreme points of the set $\mathcal{M}^{T}\left(A_{*}\right)$ (see [3, Theorem 4.4] or [12, Proposition 12.4]), and hence they are pairwise mutually singular. Invoking Lemma 5.8 gives the claim. 


\subsection{An application of Theorem 5.1}

In [9] Janusz Matkowski posed a problem asking about the existence of nonlinear monotonic and continuous solutions $\Phi:[0,1] \rightarrow \mathbb{R}$ of a very particular case of the equation

$$
\Phi(x)=\sum_{n=0}^{N} \Phi\left(f_{n}(x)\right)-\sum_{n=1}^{N} \Phi\left(f_{n}(0)\right) .
$$

Motivated by this problem, denote by $\mathcal{M}$ the vector space spanned by $\mathcal{W} \cup\{\mathbb{1}\}$, where $\mathbb{1}$ denotes the constant function that equals 1 on $[0,1]$. Note that by Theorem 5.1 and the fact that $\phi(0)=0$ for each $\phi \in \mathcal{W}$, the set $\mathcal{W} \cup\{\mathbb{1}\}$ is a basis of $\mathcal{M}$.

Proposition 5.9. Every function belonging to $\mathcal{M}$ is a continuous solution of Eq. (24). Moreover, if $\phi_{1}, \ldots, \phi_{m} \in \mathcal{W}$ and $\alpha_{1}, \ldots, \alpha_{m} \in \mathbb{R}$ are of the same sign, then the function $\sum_{i=1}^{m} \alpha_{i} \phi_{i}+\alpha_{0}$ is monotone for every $\alpha_{0} \in \mathbb{R}$.

Proof. Fix $\Phi \in \mathcal{M}$. Then there exist $\alpha_{0}, \ldots, \alpha_{m} \in \mathbb{R}$ and $\phi_{1}, \ldots, \phi_{m} \in \mathcal{W}$ such that $\Phi=\sum_{i=1}^{m} \alpha_{i} \phi_{i}+\alpha_{0}$. Obviously, $\Phi$ is continuous. According to the first assertion of Lemma 2.2 we see that $\phi_{i}\left(f_{0}(0)\right)=0$ for every $i \in\{1, \ldots, m\}$, and hence applying also (E), we obtain

$$
\begin{aligned}
\sum_{n=0}^{N} \Phi\left(f_{n}(x)\right)-\sum_{n=1}^{N} \Phi\left(f_{n}(0)\right)= & \sum_{n=0}^{N}\left(\sum_{i=1}^{m} \alpha_{i} \phi_{i}\left(f_{n}(x)\right)+\alpha_{0}\right) \\
& -\sum_{n=1}^{N}\left(\sum_{i=1}^{m} \alpha_{i} \phi_{i}\left(f_{n}(0)\right)+\alpha_{0}\right) \\
= & \sum_{i=1}^{m} \alpha_{i}\left(\sum_{n=0}^{N} \phi_{i}\left(f_{n}(x)\right)-\sum_{n=0}^{N} \phi_{i}\left(f_{n}(0)\right)\right)+\alpha_{0} \\
= & \sum_{i=1}^{m} \alpha_{i} \phi_{i}(x)+\alpha_{0}=\Phi(x)
\end{aligned}
$$

for every $x \in[0,1]$.

The moreover part of the assertion is clear.

\section{Acknowledgements}

The research of both authors was supported by the University of Silesia Mathematics Department (Iterative Functional Equations and Real Analysis program). Furthermore, the research leading to these results has received funding from the European Research Council under the European Union's Seventh Framework Programme (FP/2007-2013) / ERC Grant Agreement No. 291497 
while the second author was a postdoctoral researcher at the University of Warwick.

Open Access. This article is distributed under the terms of the Creative Commons Attribution 4.0 International License (http://creativecommons.org/licenses/by/4.0/), which permits unrestricted use, distribution, and reproduction in any medium, provided you give appropriate credit to the original author(s) and the source, provide a link to the Creative Commons license, and indicate if changes were made.

Publisher's Note Springer Nature remains neutral with regard to jurisdictional claims in published maps and institutional affiliations.

\section{References}

[1] Barnsley, M.: Fractals Everywhere. Academic Press Inc., Boston (1988)

[2] Biswas, H.R.: Ergodic theory and mixing properties. J. Pure Appl. Math. Adv. Appl. 12(1), 1-24 (2014)

[3] Einsiedler, M., Ward, T.: Ergodic Theory with a View Towards Number Theory. Graduate Texts in Mathematics, vol. 259. Springer, London (2011)

[4] Falconer, K.: Techniques in Fractal Geometry. Wiley, Chichester (1997)

[5] Hutchinson, J.E.: Fractals and self-similarity. Indiana Univ. Math. J. 30(5), 713-747 (1981)

[6] Kannan, R., Krueger, C.K.: Advanced analysis on the real line. Universitext, Springer, New York (1996)

[7] Lasota, A., Mackey, M.C.: Chaos, Fractals, and Noise. Stochastic Aspects of Dynamics. Volume 97 of Applied Mathematical Sciences, 2nd edn. Springer, New York (1994)

[8] Lasota, A., Myjak, J.: Generic properties of fractal measures. Bull. Polish Acad. Sci. Math. 42(4), 283-296 (1994)

[9] Matkowski, J.: Remark on BV-solutions of a functional equation connected with invariant measures. Aequ. Math. 29(2-3), 210-213 (1985)

[10] Morawiec, J., Zürcher, T.: Attractor of cantor type with positive measure. Results Math. 73(2), 1-13 (2018)

[11] Morawiec, J., Zürcher, T.: On a problem of Janusz Matkowski and Jacek Wesołowski. Aequ. Math. 92(4), 601-615 (2018)

[12] Phelps, R.R.: Lectures on Choquet's Theorem. Volume 1757 of Lecture Notes in Mathematics, 2nd edn. Springer, Berlin (2001)

[13] Wise, G.L., Hall, E.B.: Counterexamples in Probability and Real Analysis. The Clarendon Press, Oxford University Press, New York (1993)

Janusz Morawiec and Thomas Zürcher

Instytut Matematyki

Uniwersytet Śląski

Bankowa 14

40-007 Katowice

Poland

e-mail: morawiec@math.us.edu.pl

Thomas Zürcher

e-mail: thomas.zurcher@us.edu.pl

Received: January 23, 2018 